\title{
Collaborative Editing for All: The Google Docs Example
}

\author{
Giulio Mori ${ }^{1}$, Maria Claudia Buzzi ${ }^{1}$, Marina Buzzi ${ }^{1}$, \\ Barbara Leporini ${ }^{2}$, and Victor M. R. Penichet ${ }^{3}$ \\ ${ }^{1}$ CNR-IIT, via Moruzzi 1, 56124 Pisa, Italy \\ \{Giulio.Mori, Claudia.Buzzi,Marina.Buzzi\}@iit.cnr.it \\ ${ }^{2}$ CNR-ISTI, via Moruzzi 1, 56124 Pisa, Italy \\ Barbara.Leporini@isti.cnr.it \\ ${ }^{3}$ Computer Systems Department, University of Castilla-La Mancha, Albacete, Spain \\ victor.penichet@uclm.es
}

\begin{abstract}
Collaborative software tools allow people to share documents and knowledge via Internet, in a simple, economic and efficient way. Unfortunately collaborative software often relies heavily on visual features and dynamic technologies with user interfaces that are difficult to use via screen reader, or are sometimes even inaccessible for the blind.

In this paper we illustrate and discuss results of an accessibility inspection of the main collaborative functions of Google Docs using the JAWS screen reader. Results highlight several difficulties encountered when interacting with elements of the Google Docs interfaces. Content perception is often incomplete, since many elements or changes occurring in the collaborative environment are not intercepted by the screen reader and announced to the user. In addition, the behavior of the collaborative functions analyzed (as well as the rendering) changes from one web browser to another. Some general guidelines are discussed, for designing user interfaces of collaborative editors that are more usable when interacting via screen reader.
\end{abstract}

Keywords: groupware, collaborative editing, accessibility, blind, screen reader.

\section{Introduction}

Collaborative editing tools allow people to work together via Internet in a simple, economic and efficient way. However, their user interfaces are not always easy to use, nor do they consider the needs of differently-abled persons, thus excluding a considerable number of potential users.

To create a valuable product, both accessibility and usability must be considered in the design phase. Accessibility is a prerequisite that permits users to perceive online content and interact, while usability enhances the quality of the interaction, which should be simple, efficient and satisfying. Studying principles of web design to allow easy Internet use for each type of disability is difficult, since disabilities are numerous and heterogeneous. Nevertheless, many user studies suggest that totally blind users encounter more difficulty than people with other sensory disabilities (such as low vision, or motor or hearing impairment) when executing specific tasks [1], [2], [3], so we focus our research on them. 
Blind people usually interact with computers via screen reader, voice synthesizer and keyboard. The sequential interaction may lead to serious problems, such as content serialization and information overload. The screen reader adds a level of complexity to the interaction, when designing for blind users it is essential to consider the overall interaction, involving the perceptual, motor and cognitive systems of the Human Processor Model [4].

In this paper we specifically analyze several important collaborative features when interacting via screen reader. Unfortunately, collaborative tools mostly use visual techniques to provide information (e.g., who is working simultaneously on the system, what parts are being modified, etc.). In this context, we take into account the main features of collaborative tools in order to understand how they could be made more usable for blind users. Specifically, we consider the collaborative features available on an editing tool by analyzing the popular Google Docs (http://docs.google.com) as a collaborative editing system. At the beginning of our study we analyzed the main accessibility problems of the Google Docs environment when a blind user interacts via screen reader and voice synthesizer [5]. In this paper we specifically discuss accessibility and usability issues for collaborative functions available in Google Docs. Typical collaborative features of groupware environments include collaboration, cooperation, coordination, communication, information sharing, awareness, time and space.

To verify the accessibility of typical groupware, we inspected specific functions of Google Docs involving interactions with other persons [6]: inviting people, getting a link to share, seeing who has access, email with attachment, sending messages to collaborators, publishing a web page, uploading files, reviewing history and comments. The test aimed to evaluate the degree of accessibility of collaborative features of Google Docs when interacting via screen reader (we used JAWS, the most commonly used screen reader in the Italian blind community [7]), and was performed by the three sighted authors of these paper (with computer screen turned off), and by the blind author of this study, who is proficient in using JAWS.

Our results offer an overview of several difficulties encountered when interacting with elements of the Google Docs interfaces. Content perception is often incomplete, since many elements or changes occurring in the collaborative environment are not announced by the screen reader. As an additional problem, the functioning (and rendering) of the collaborative functionalities analyzed could differ depending on the web browser used. Finally, we will suggest some basic guidelines for designing more effective, efficient and satisfactory UIs for collaborative editing.

\section{Accessibility and Usability of Google Docs Collaborative Features}

In this section we only analyze specific functions of Google Docs that involve collaborative interactions [6]. The degree of accessibility of these functions have been verified using the screen reader JAWS (versions 10 and 12), and the Internet Explorer 8 and Firefox 3.6.13 browsers. Interaction with these two browsers has shown significantly different behaviors that may negatively impact on the user. In the following the issues encountered for each main collaborative function will be described. 


\subsection{Invite People}

Considering the main interface of Google Documents shown in Fig. 1, it is possible to share a document with other people in three ways:

- Accessing the "share" pull-down menu of the main interface (Fig. 1);

- Selecting the checkbox associated to a document and accessing the "share" item menu, which appears after pressing the simulating "right mouse button" key on the keyboard (Figure 1);

- Opening a document and accessing the "share" pull-down menu from the editor (Fig. 2).

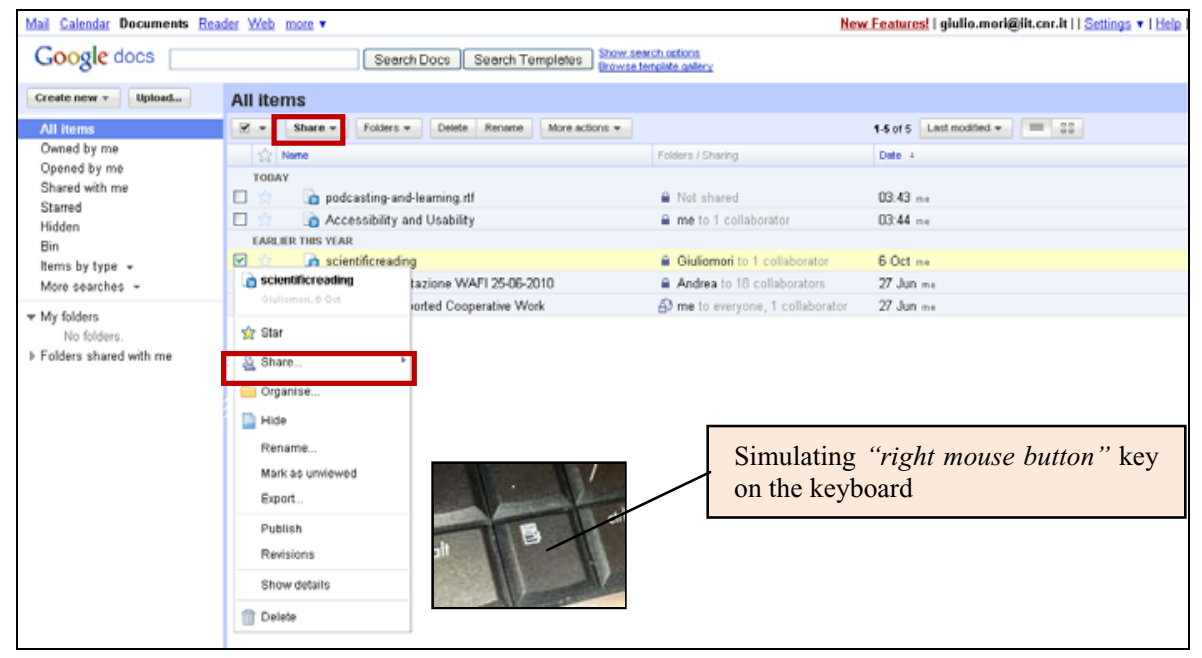

Fig. 1. Main interface of Google Docs - Documents

Verifying the accessibility of these three modalities for inviting people we observed:

1) The "share" pull-down menu is not accessible on both Internet Explorer and Firefox. 2) The checkbox which is associated with a document is only selectable on Firefox. The checkbox is not selectable using Internet Explorer, so it is not possible to access the menu functions related to a selected document using that browser. In terms of usability, the association between a checkbox and a document is not intuitively perceived by a user interacting via screen reader and keyboard.

Besides, 3) the "share" pull-down menu on the editor interface is not very accessible (after pressing the ESC key in the editing area), because the pull-down menu is announced as a general "button menu". Interaction improves a little using JAWS v. 12. Selecting the "share" pull down or item menu it would open the "sharing settings" window (Fig. 3a) where it is possible to insert collaborators' addresses, but this only happens using Firefox. 
This page also presents some accessibility problems: a) many labels of the elements (for example "Add people", etc.) are not announced by the screen reader, so the blind user cannot know what information to insert in that field; b) sometimes we detected the loss of the window focus during interaction when the "Sharing Settings" window is opened; c) in case no people are added, the "Share" button is correctly non-clickable, but after closing the "sharing settings" window (using the "Cancel" button), a warning message (Fig. 3b) appears and it is not announced by the screen reader; d) after closing the window for sharing a document, focus goes to the "mail" link, on the top of the main interface (Fig. 1), probably provoking user frustration.

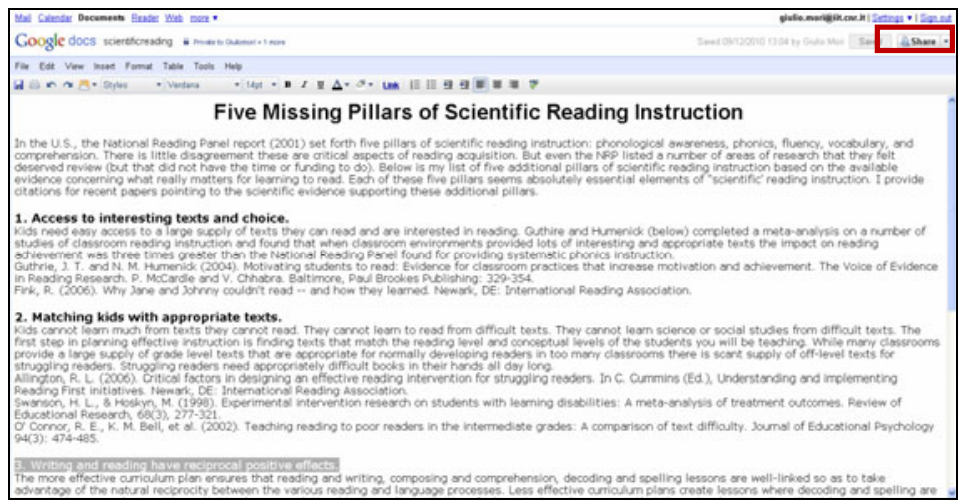

Fig. 2. Editor interface of Google Documents

(a)

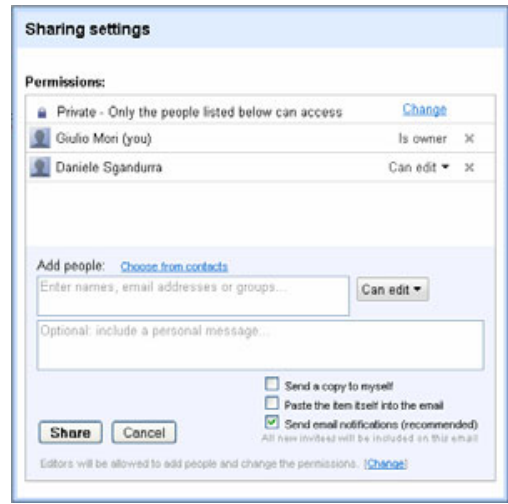

Please specify at least one recipient.

(b)

Fig. 3. a) The sharing settings window; b) No added people warning message

\subsection{Get the Link to Share/Email as Attachment/Send Message to Collaborators}

These functions are not accessible through the "share" pull-down menu (Fig. 1) both on Internet Explorer and Firefox. On the contrary, the share functionalities are accessible only using Firefox pressing the key simulating the right mouse button 
(after the selection of the associated checkbox to a document - Fig. 1), or using the "Share" pull down menu on the editor (Fig. 2).

The document can be shared with other collaborators sending an email (message) containing the link to the document (Fig. 4b) or an email containing the document as an attachment (Fig. 4a). These window interfaces are reachable on Firefox but present these issues (Fig. 3a): a) labels are not announced by the screen reader; b) windows sometimes lose focus; c) warning message (Fig. 4c) when no email addresses are inserted is not announced; d) after closing the "Email as Attachment" and "Send messages" windows (Fig. 4a, 4b), focus goes to the "mail" link, on the top of the main interface (Fig. 1).

Our test interacting via JAWS screen reader and keyboard emphasized some difficulties inserting email addresses on the "Email as Attachment" window.

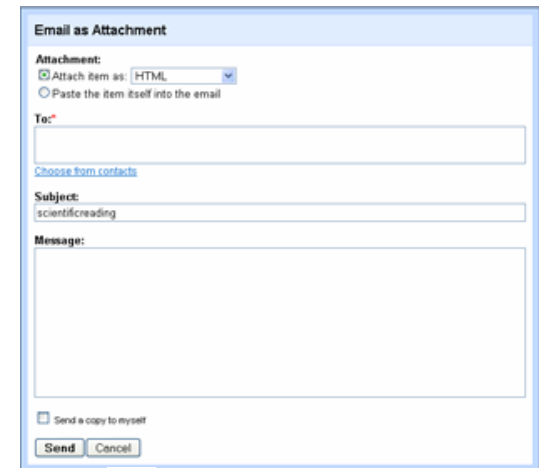

(a)

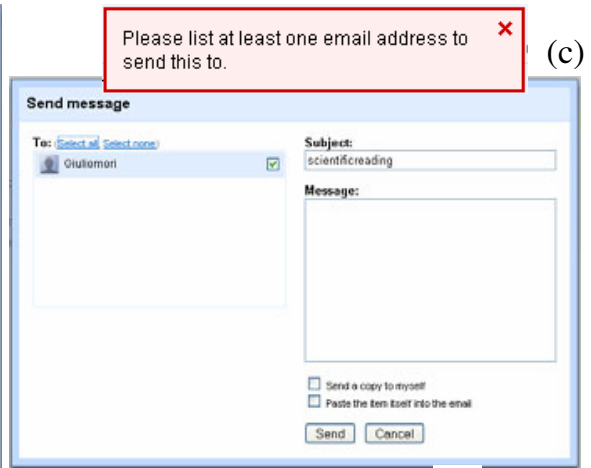

(b)

Fig. 4. a) Email as Attachment window; b) Send message window; c) warning message

\subsection{See Who Is Accessing}

Considering the editor, the screen reader is not able to perceive who is working at the same time on the same document, as appears visually (Fig. 5). Sometimes, when two or more collaborators are modifying the same part of a document, a warning message appears (Fig. 6). This message is accessible, but user awareness (i.e. a user's knowledge about the actions that other users are performing in the system) depends on whether the focus is on the window.

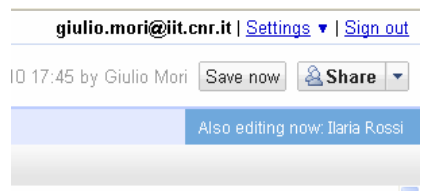

Fig. 5. Feedback about who is accessing 


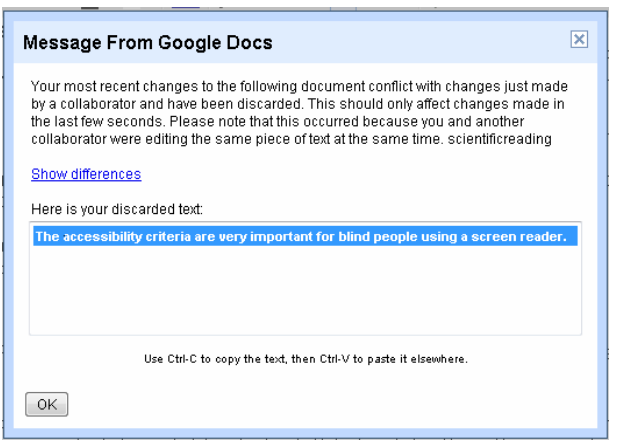

Fig. 6. Warning for collaborators modifying the same part of a document

\subsection{Publish as Web Page}

This function is reachable only using Firefox, selecting the check box associated with a document and pressing the key simulating the right mouse button (Fig. 1). Interactive elements of the publishing interface are accessible, but text is not automatically announced (Fig. 7).

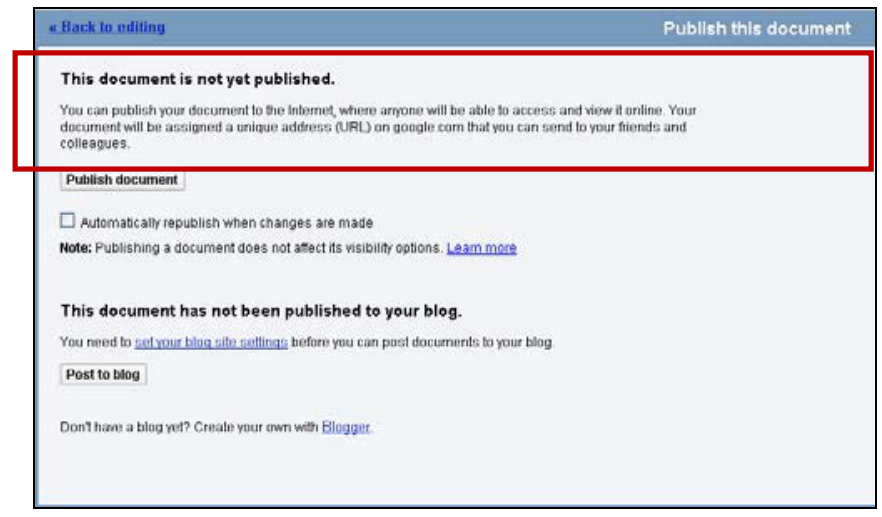

Fig. 7. Publishing interface

\subsection{Upload}

The "Upload" function is not accessible using JAWS v. 10. Using Internet Explorer the button on the main interface (Fig. 1) is announced, but it is not clickable; with Firefox the button is not announced and then is not clickable. In contrast, using JAWS v. 12, the "Upload" function is accessible. However, after accessing the upload interface (Fig. 8), the "Select files to upload" link is only accessible using Firefox (although it is announced as "Browser"). The "Start upload" button is accessible with both browsers (although it is useless on Internet Explorer, due to the inaccessibility of the "Select files to upload" link). 


\subsection{See Revision History}

This function is reachable only using Firefox, in three ways: 1) selecting the check box associated to a document and pressing the key simulating the right mouse button (Fig. 1), 2) after the selection of the checkbox, through the "More actions" pulldown (difficult for the user), 3) in the editor by means of the menu "File" (although it works only with JAWS v. 12), which is very hard to access (Fig. 9).

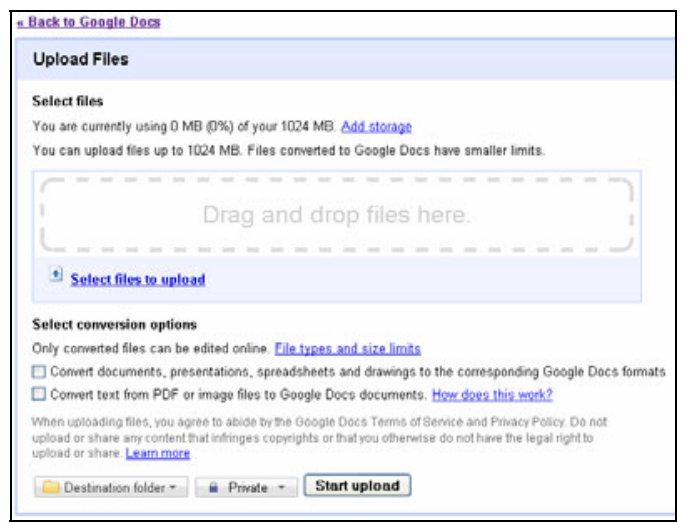

Fig. 8. Upload interface

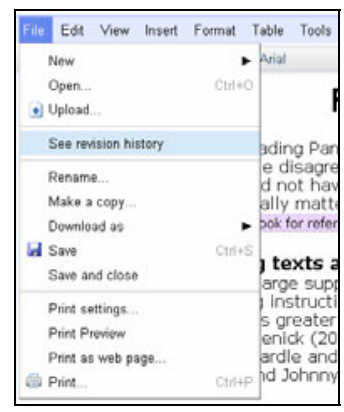

Fig. 9. Revision History interface

Looking at the "Revision History" interface (Fig. 10a), links of different revisions are accessible, but the text of each revision is not announced (also exploring via arrow keys). The revision item on the menu appears only after selecting the checkbox of one document. The "Compare Ticked" button allows to compare two or more different versions of the same document, but the use of this functionality is not simple, because the user must make the extra effort to select at least two checkboxes (each one associated with a revision), and then reach back sequentially to the "Compare Ticked" button. Besides, after pressing the "Compare Ticked" button, the revision differences are not announced by the screen reader (Fig. 10b).
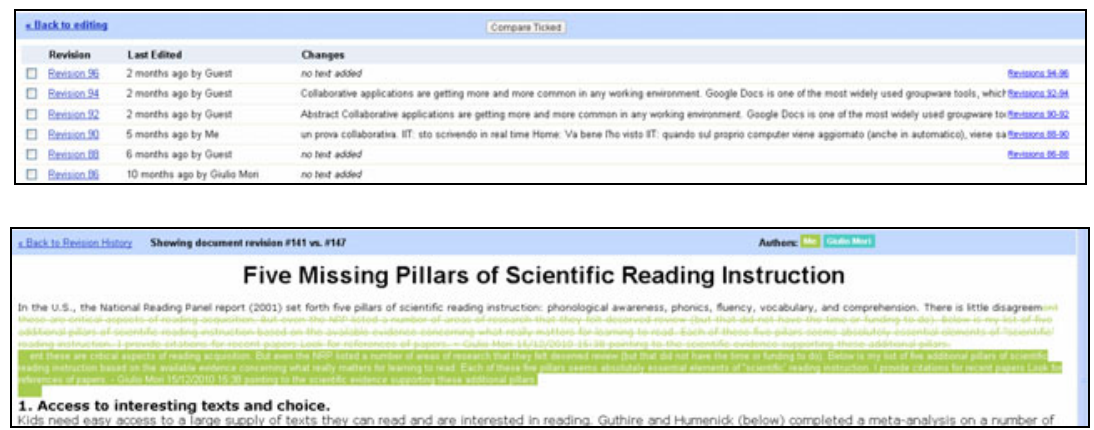

Fig. 10. a) Revision History interface; b) Compare Ticked interface 


\subsection{Comment}

A comment can be inserted into a document only using the Firefox browser, through the "Comment" item of the "Insert" menu in the editor page (hard to access), so this task is quite difficult to complete for a blind user (Fig.11).

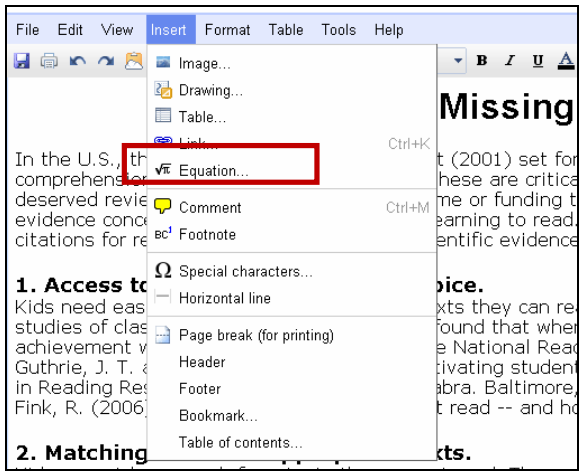

Fig. 11. Not accessible (via screen reader) to insert "Comment" item

However, although the "Comment" function is not easy to complete, as a test we forced the comment, observing that it is not possible to exit from the comment field using the keyboard (Fig. 12). Besides, a blind user cannot understand the difference between a comment and the text contained in the document, since the screen reader does not announce any difference, and it reads it in the same way (Fig. 12).

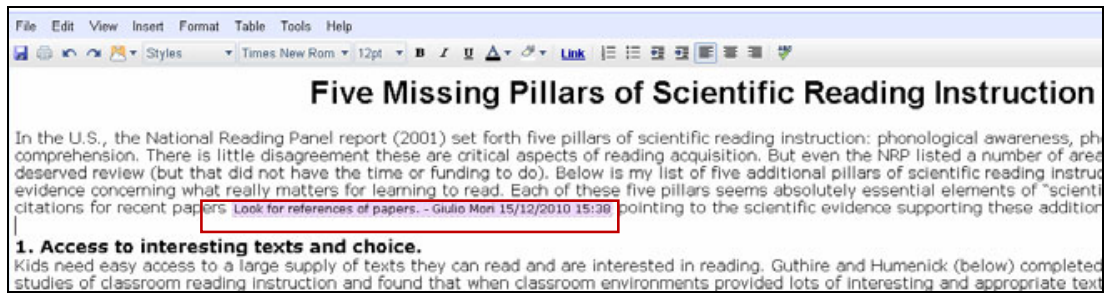

Fig. 12. A comment into a document

\section{Some Suggestions for Solving Accessibility Problems}

Making the important features and properties in a tool for collaborative editing accessible and usable for blind users presents various peculiarities. A previous study [5] highlighted possible problems arising when accessing Google Docs and writing a document. In this paper, more specific aspects of the collaborative functions available in document sharing are described. Based on the examined case study we suggest a few specific aspects to be keep in mind when thinking of more detailed guidelines or criteria for a more usable editing environment. These criteria should be applied in 
addition to the general W3C guidelines for Web Content Accessibility [8]. In brief, when developing a collaborative editing environment, it is important to make:

- Editing operable - Main editing functions must be operable via keyboard. The edited text has to be readable char by char, word by word, and line by line using an editing cursor. Selection should be possible through the standard operating system shortcuts (e.g. Ctrl+Shift+arrow keys) and highlighted by using standard color combinations (i.e. while conserving the compatibility with the assistive technologies). The focus should be easily switchable between the main areas, such as editing, toolbars and any other menu or interactive elements available for important functions (e.g. Save, Close, etc.). Opening the editor in a new window could greatly improve usability via screen reader.

- Awareness perceivable - Information on other connected users should be provided through a suitable method in order to appropriately provide the information to the assistive technologies. Status and actions concerning other collaborators should be made accessible to the assistive technology. Information on who is online or offline, or what they are doing, should be available and easy to obtain at any time.

- Co-editing understandable - When two or more users are working on the same document together, information on portions that are free to be edited, on content blocks that are being edited by other users at the same time, and on which parts have been modified, should be adequately provided to the assistive technology so that the user can quickly understand.

- Dynamic messages and instantaneous feedback - Feedback and short messages or alerts should be made clear and easy to read. Alerting should be promptly provided to the assistive technology so that users can be readily informed. Short sounds or audio feedback could improve user perception of given events, such as failure or success, or outcomes for specific performing results (e.g., a sound for bold style application, another one for italics, and so on).

Based on these principles to consider, specific guidelines could be better detailed. ARIA-suite, the W3C-WAI Accessible Rich Internet Applications Suite [9], a valuable technical support for implementing more accessible and usable UIs, would also be applicable to collaborative editing environments.

\section{Discussion}

Collaborative tools are increasingly adopted in environments such as working group activities, e-learning systems, distance education and social networks. Collaboration is important, and increases our opportunities: reinforcing our knowledge, learning more, sharing ideas, getting feedback. For this reasons user interfaces of collaborative software applications should be accessible and usable for all.

In this paper, we have shown issues of interacting via screen reader with Google Docs user interfaces, focusing only on its collaborative features. Manual inspection with the JAWS screen reader has highlighted some major accessibility issues with Internet Explorer, but Firefox also presents some usability deficits. The latest version of JAWS offers benefits when using Firefox, allowing more satisfying interaction for 
the blind, but JAWS is a commercial product so not all blind users can update immediately to the latest version. Additional effort is required to guarantee easy, effective and satisfying access via screen reader, especially from Google application designers and software engineering, for implementing accessible and usable user interfaces, conforming to the W3C WAI Web Content Accessibility Guidelines v.2.0 and WAI-ARIA.

Last, some basic suggestions have been offered in order to make collaborative environments more useful for blind users. The progress of collaborative environment usability is valuable in general for all users, and the benefits of accessibility will be reflected in the wider diffusion and use of these kinds of tools and applications.

\section{References}

1. Craven, J., Brophy, P.: Non-visual access to the digital library: the use of digital library interfaces by blind and visually impaired people. Technical report, Manchester: Centre for Research in Library and Information Management - CERLIM (2003),

http: / / www. cerlim.ac.uk/pubs / index.php

2. Ivory, M. Y., Yu, S., Gronemyer, K.: Search result exploration: a preliminary study of blind and sighted users' decision making and performance. In: Extended abstracts of CHI 2004, pp. 453-456 (2004)

3. Petrie, H., Hamilton, F., King, N.: Tension, what tension?: Website accessibility and visual design. In: Proc. 2004 International Cross-disciplinary Workshop on Web Accessibility (W4A), pp. 13-18 (2004)

4. Card, S.K., Moran, T.P., Newell, A.: The Psychology of Human-computer Interaction, pp. 29-97. Lawrence Erlbaum Associates, London (1983)

5. Buzzi, M.C., Buzzi, M., Leporini, B., Mori, G., Penichet, V.M.R.: Accessing Google Docs via Screen Reader. In: Miesenberger, K., Klaus, J., Zagler, W., Karshmer, A. (eds.) ICCHP 2010. LNCS, vol. 6179, pp. 92-99. Springer, Heidelberg (2010)

6. Garrido, J.E., Penichet, V.M.R., Lozano, M.D., Buzzi, M.C., Buzzi, M., Leporini, B., Mori, G.: Analysing Google Documents as groupware tool and an improvement proposal. In: XI Congreso Internacional de Interacción Persona-Ordenador (2010)

7. Leporini, B., Andronico, P., Buzzi, M., Castillo, C.: Evaluating a modified Google user interface via screen reader. The Universal Access in the Information Society (UAIS) 7(3), $155-175$ (2008)

8. W3C. Web Content Accessibility Guidelines 2.0. (December 11, 2008), http: / / www.w3 . org/TR/WCAG2 0 /

9. W3C. WAI-ARIA Technical Specification, http://www.w3 .org/TR/wai-aria/ 